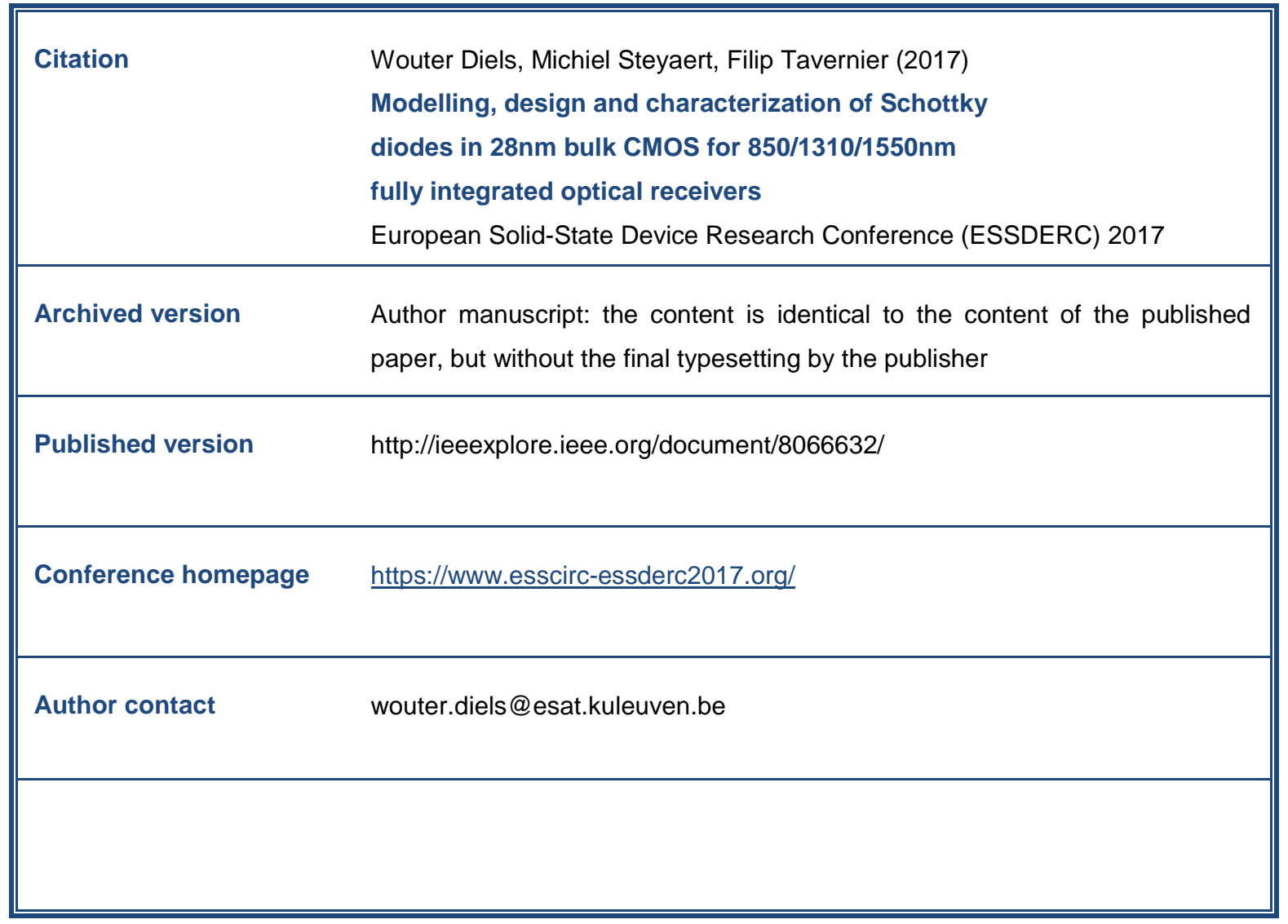

(article begins on next page) 


\title{
Modelling, design and characterization of Schottky diodes in 28nm bulk CMOS for 850/1310/1550nm fully integrated optical receivers
}

\author{
Wouter Diels, Michiel Steyaert and Filip Tavernier \\ Department of Electrical Engineering, Katholieke Universiteit Leuven \\ 3001 Leuven, Belgium
}

\begin{abstract}
This paper presents N-well Schottky diodes for high speed optical detection in 28nm CMOS technology. These diodes enable fully integrated CMOS optical receivers suited for the 850,1310 and $1550 \mathrm{~nm}$ telecommunication bands. The measured 1310 and $1550 \mathrm{~nm}$ DC responsivity is $0.71 \mathrm{~mA} / \mathrm{W}$ and $0.16 \mathrm{~mA} / \mathrm{W}$ respectively at $1.5 \mathrm{~V}$ reverse bias when backside illumination is performed while the $850 \mathrm{~nm}$ responsivity is $0.27 \mathrm{~mA} / \mathrm{W}$ at the same biasing when frontside illumination is done. This is the first reported CMOS photodetector demonstrated at these three wavelengths. The measured capacitance-to-area ratio at zero bias is $1.7 \mathrm{mF} / \mathrm{m}^{2}$.
\end{abstract}

\section{INTRODUCTION}

Glass fiber-optic communication links have nearly unrivalled performance concerning data rates and distance. These links generally make use of 1310 and 1550nm light trough a single-mode fiber (SMF) or $850 \mathrm{~nm}$ light through a multimode fiber (MMF). At the receiving end, the optical receiver typically consists of a high-speed photodetector to convert the optical signal into an electrical current, a transimpedance amplifier (TIA) to convert this current into a voltage and a post-amplifier to amplify this voltage to rail-to-rail levels [1]. As the photodetector is the first block of the receiver, its performance largely determines the overall performance of the full receiver.

For high-end receivers, each of these blocks is typically implemented on a separate chip in a different technology. The photodetectors of these systems are either PiN- or avalanche photodiodes. These diodes are fabricated in dedicated technologies to obtain high responsivity, high bandwidth and low capacitance for low dark currents. While these receivers have excellent performance, this multi-chip implementation and the resulting stringent packaging requirements result in a high cost. On the other hand, Photonic integrated circuits (PICs), keep gaining more attention [2], since these technologies enable integrating optical components, such as lasers, electro-optical modulators and photodetectors, on a single die. However, while CMOS-integrated photonics technologies have been demonstrated [3], most of them make use of relatively old CMOS technologies. Furthermore there is a lack of standardization.

Optical receivers can also be monolithically integrated in (modern) CMOS technologies. However, because there is barely any light absorption in silicon for wavelengths longer than $1.1 \mu \mathrm{m}$, nearly all research has been aimed at $850 \mathrm{~nm}$ receivers. As PiN-photodiodes are not available in standard CMOS technologies, the reported optical receivers make use of PN-diodes, some in the linear region [4], [5], others in the avalanche region [6]. P-substrate/ $\mathrm{N}$-well junctions provide the largest space charge region (SCR) and smallest capacitance and are thus the preferred choice for photodiodes in the linear region. Although the DC responsivity for 850nm light is relatively high, the low absorption coefficient of silicon results in a low photocurrent bandwidth (1-10MHz) because of carriers generated deep in the substrate [7]. An additional equalizer is necessary to compensate for this intrinsic low bandwidth. Alternatively, spatially modulated light (SML) photodiodes provide bandwidth extension at the cost of reduced responsivity, but ultimately degrade the signal-to-noise ratio (SNR) since half of the light is reflected by the metal coverage. When the reverse bias voltage is increased to high voltages, PN-junctions go into avalanche region. Operation in this region leads to increased responsivity and bandwidth, but also to larger photodiode noise. As junctions with thin avalanche regions result in the lowest excess noise factor [8], $\mathrm{P}+/ \mathrm{N}$-well(/P-substrate) junctions are the preferred choice for avalanche photodiodes in CMOS.

As mentioned, PN-photodiode-based receivers in CMOS are only suited for short wavelength links $(660 \mathrm{~nm}$ in plastic optical fiber or $850 \mathrm{~nm}$ in multi-mode fiber). There are thus no CMOS receivers reported for 1310/1550nm SMF links, which are traditionally used for long-haul communication. In [9], N- and P-well Schottky diodes in 40nm CMOS are presented, which can convert 1310nm light through internal photoemission (IPE). These devices are however not optimized for fiber coupled receivers: the large rectangular shape leads to excessive and unnecessary capacitance, while the distributed layout leads to a low diode fill factor, which results in a low responsivity. In this work, two circular plain layout $\mathrm{N}$-well Schottky photodiodes in $28 \mathrm{~nm}$ CMOS are presented. Their sizes are matched to a SMF and a MMF.

This paper is structured as follows. Section II gives some insight of the mechanisms of Schottky photodiodes, as well as design and layout considerations. In Section III, the I-V, capacitance and responsivity measurement results of the diodes are presented while comparing them to the N-well diode in [9]. Finally, Section IV gives a conclusion. 


\section{CMOS SCHOTTKY DIODES}

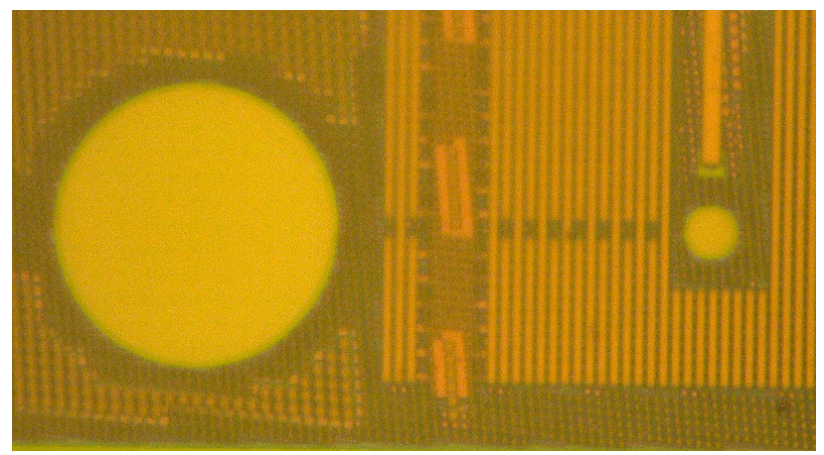

Fig. 1: Zoomed-in photograph of the presented 28nm Schottky diodes. The diode on the left is matched to a MMF, the diode on the right to a SMF.

A photograph of the presented diodes can be seen in Fig. 1. In Fig. 2, the working principle of internal photoemission (IPE) for an N-type Schottky diode can be seen. The light gets absorbed in the metal and if the excited electron has sufficient energy, it can cross the Schottky barrier $\phi_{B}$. The maximum detectable wavelength is thus given by $h c / q \phi_{B}$, where $\mathrm{h}$ is Planck's constant, $\mathrm{c}$ is the speed of light and $\mathrm{q}$ is the elementary charge. This implies $1550 \mathrm{~nm}$ and $1310 \mathrm{~nm}$ can be detected for a Schottky barrier of $0.8 \mathrm{eV}$ and $0.95 \mathrm{eV}$ respectively. The quantum effiency $(\mathrm{QE})$ is however rather low, since the metal reflects most of the light. The closer the photons are absorbed to the barrier, the higher the probability to cross the barrier. The QE also increases for bigger energy differences between photon energy and $\phi_{B}$ [10].

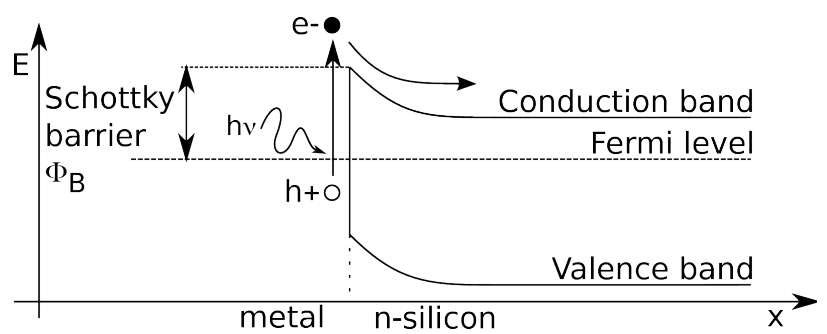

Fig. 2: The principle of internal photoemission: a photon gets absorbed in the metal, exciting an electron to a higher energy level in the process. If the electron has gained sufficient energy, it can cross the Schottky barrier $\phi_{B}$.

A topside and cross section view of the presented diodes can be seen in Fig. 3. Schottky diodes can be realized in CMOS by placing contacts to the relatively lowly doped N-well. A wide junction is formed between the silicon and the silicide. Ohmic contacts to the highly doped $\mathrm{N}+$ layer make up the cathode of the device. Both diodes are circular and have a diameter of $51.3 \mu \mathrm{m}$ and $9 \mu \mathrm{m}$. These dimensions are chosen as to be matched to MMFs and SMFs, of which the core typically has a diameter of $50-62.5 \mu \mathrm{m}$ and $8-10 \mu \mathrm{m}$ respectively. Light can be impinged from the top (frontside illumination - FSI) or from the back (backside illumination - BSI). FSI can be performed for the three telecom wavelengths, but only $1310 \mathrm{~nm}$ and $1550 \mathrm{~nm}$ can be impinged from the backside due to the non-transparency of silicon for $850 \mathrm{~nm}$ light. When the chip is illuminated from the top, the photons get absorbed at the top of the M1 layer. The excited electrons must then travel a relatively large distance without losing energy in order to cross the barrier. The probability of these electrons crossing the barrier is much lower than those generated close to the junction, as is the case for BSI. Because of this, BSI results in larger responsivity than FSI. The intrinsic bandwidth of IPEbased photocurrent can easily be in the $\mathrm{GHz}$ range: unlike 850nm FSI for CMOS PN-diodes, all carriers are generated very locally and the intrinsic bandwidth is determined by the short transit time to cross the SCR.

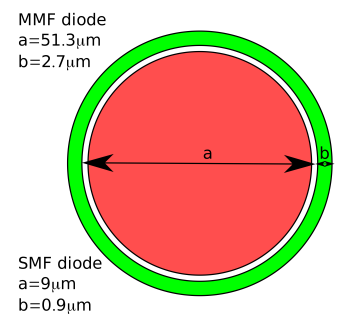

(a)

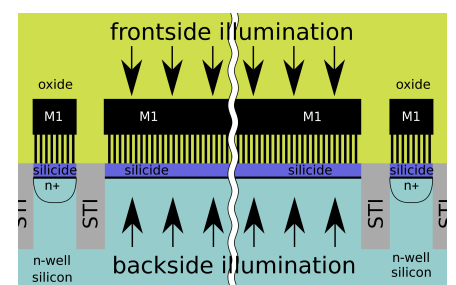

(b)
Fig. 3: (a) topside view - red region denotes Schottky regions, green region Ohmic regions (b) cross-section view - silicide and $\mathrm{N}$-well silicon form Schottky region, silicide and $\mathrm{N}+$ region form Ohmic region

The chosen layout maximizes the realizable diode area and responsivity. Whereas the silicide and M1 layers make up the entire area, technology design rules limit the contact width and spacing, resulting in a maximum $10 \%$ of the device area covered with contacts. Unlike the diodes in [9], the Schottky photodiodes in this work do not have metal dummies on top of them, which means FSI can be performed on them. This opens up the possibility of $850 \mathrm{~nm}$ detection.

\section{MeAsurements}

No standard CMOS process is intended to realize Schottky diodes and thus no model is available. Therefore, the performance of the Schottky diodes is first characterized, after which an analog front-end can be designed. The equivalent schematic of the Schottky photodiode can be seen in Fig. 4. The actual junction can be represented by a voltage dependent resistor rd, a voltage dependent junction capacitance $\mathrm{Cd}$ and a photosensitive current source Iph. The series resistance Rs represents the path from the cathode to the junction. 


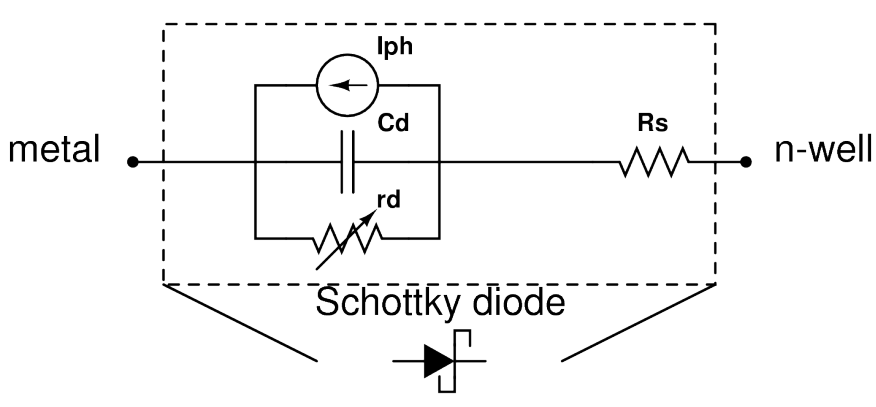

Fig. 4: Schottky photodiode equivalent schematic

\section{A. I-V characteristics}

The current-to-voltage characteristics without illumination of the devices, measured using a Keithley 2600 SMU, are shown in Fig. 5. Four important properties can be extracted from these dark current curves: the noise, the junction smallsignal resistance rd, the series resistance Rs and the Schottky barrier $\phi_{B}$.

The diode noise is dominated by the shot noise. An expression is given by (1), where $I_{p h}$ is the DC photocurrent, $I_{\text {dark }}$ is the DC dark current and BW is the extrinsic bandwidth of the diode.

$$
I_{n}=\sqrt{2 q\left(I_{p h}+I_{d a r k}\right) B W}
$$

As can be seen, the dark current of the two diodes is not larger than a few nA, suggesting a high Schottky barrier. The integrated shot noise current for $1 \mathrm{nA}$ dark current, 500nA photocurrent and $10 \mathrm{GHz}$ bandwidth is $40 \mathrm{nA}$. As the inputreferred noise current of a typical TIA at this bandwidth is much larger [5], this shot noise is of no further concern.

The diode small-signal resistance for reverse bias voltages ranges between $0.1-1 G \Omega$.

The I-V characteristics on a linear scale reveal the series resistance of the MMF and SMF diodes. They are $53 \Omega$ and $102 \Omega$ respectively. Despite the smaller device size, the series resistance of the SMF diode is larger than that of the MMF diode. This is due to the smaller spacing of the shallow trench isolation (STI), leaving a narrower path for the electrons to traverse. This series resistance due to STI could be minimized by adapting a poly-gate seperated layout as in [11]. Finally, thermionic current theory [10] reveals a zero-bias Schottky barrier $\phi_{B}$ of $0.7 \mathrm{eV}$. In comparison with the $\mathrm{N}$-well diode in [9], which has a zero-bias Schottky barrier of $0.55 \mathrm{eV}$, these diodes have a higher Schottky barrier, which results in a lower current. However, as shown in the next subsection, this also results in a lower responsivity.

\section{B. Light responsivity}

FSI was performed on a wire bonded chip with 850,1310 and $1550 \mathrm{~nm}$ light. The resulting responsivities at different biasing voltages can be seen in Fig. 6. As predicted in section II, the reponsivity increases for shorter wavelengths. The responsivity also increases for increasing reverse bias voltages

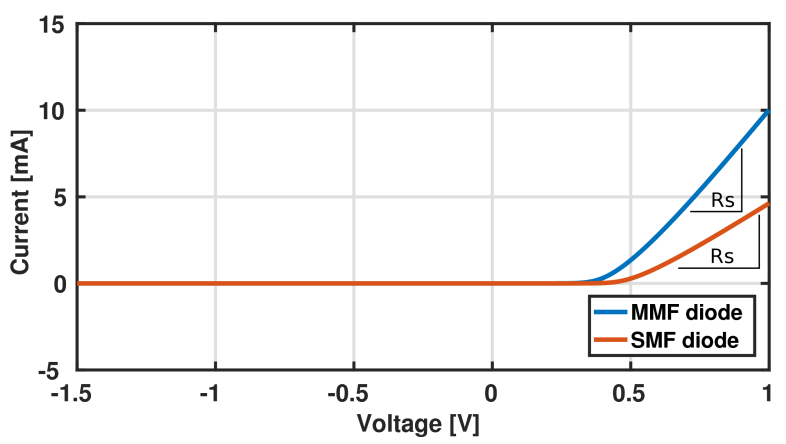

(a)

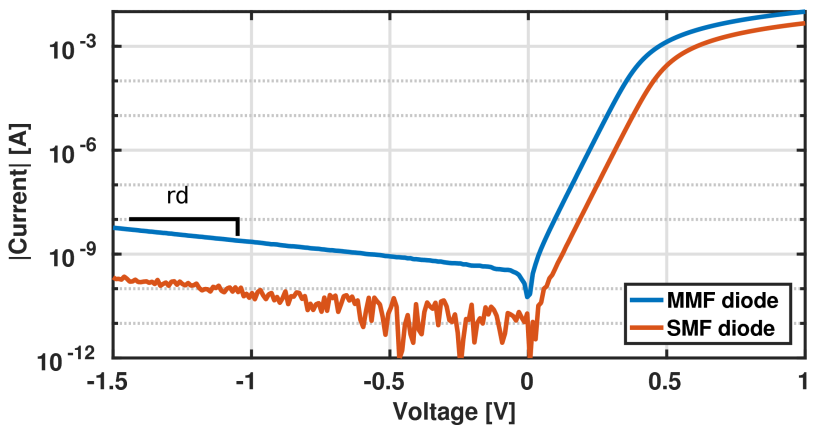

(b)

Fig. 5: IV-characteristics plotted (a) linearly (b) logarithmically

due to the barrier lowering effect [10]. At $1.5 \mathrm{~V}$ reverse bias, the diodes have a responsivity of $0.27 \mathrm{~mA} / \mathrm{W}, 5.8 \mu \mathrm{A} / \mathrm{W}$ and $1.1 \mu \mathrm{A} / \mathrm{W}$ for 850,1310 and $1550 \mathrm{~nm}$ light respectively.

For the BSI measurements, a chip was mounted on a PCB by making use of flip-chip bonding. This time, only the responsivities for 1310 and $1550 \mathrm{~nm}$ light were measured. They are shown in Fig. 7. At 1.5 reverse bias, the diodes have a responsivity of $0.71 \mathrm{~mA} / \mathrm{W}$ and $0.16 \mathrm{~mA} / \mathrm{W}$ for 1310 and $1550 \mathrm{~nm}$ light respectively. Although the fill factor of these diodes is much bigger (3.5x for diode junctions, $2.7 \mathrm{x}$ for contacts) than that of the diode in [9], the BSI 1310nm responsivity is only $1.7 \mathrm{x}$ times larger. This can again be explained by the higher Schottky barrier in this technology.

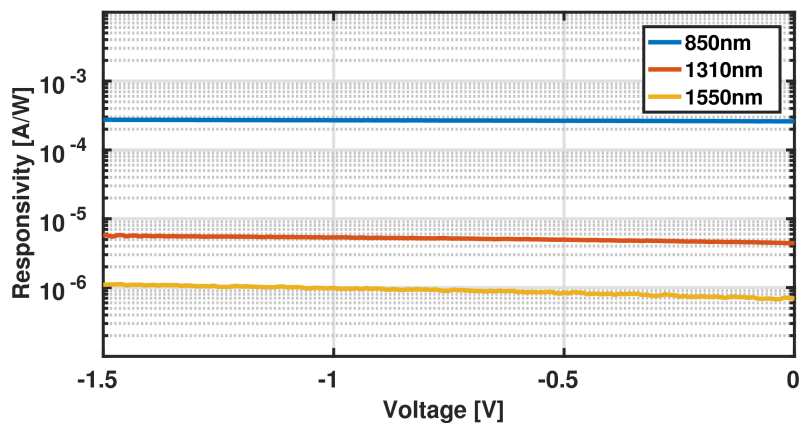

Fig. 6: Schottky photodiode responsivity for FSI with different wavelengths 


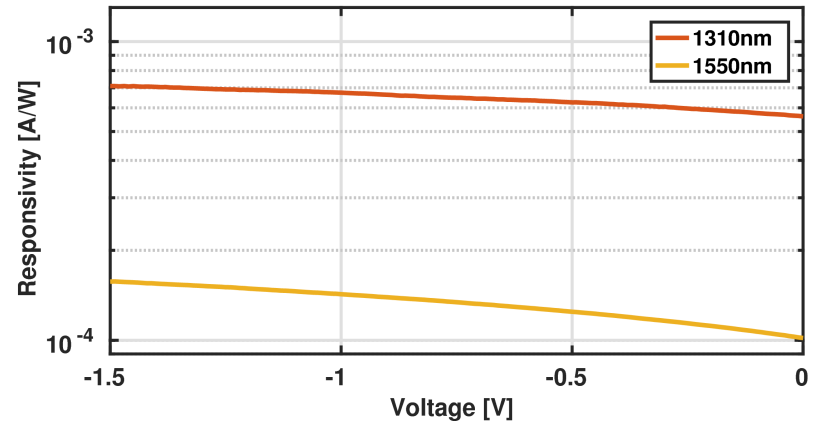

Fig. 7: Schottky photodiode responsivity for BSI with different wavelengths

\section{Capacitance extraction}

The diode small-signal impedance consists of rd in parallel with $\mathrm{Cd}$. From a certain frequency on, the junction impedance is dominated by the junction capacitance. This small-signal impedance can be obtained through s-parameter measurements. The measurements were performed with a HP $8573 \mathrm{~V}$ VNA. The resulting impedances and capacitances can be seen in Fig. 8.

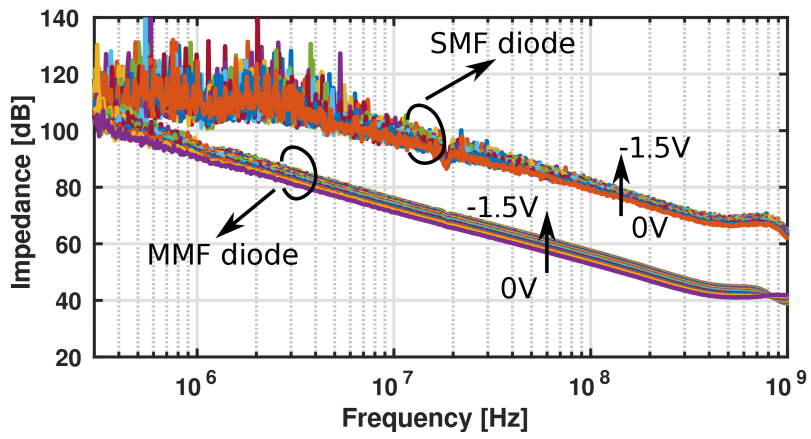

(a)

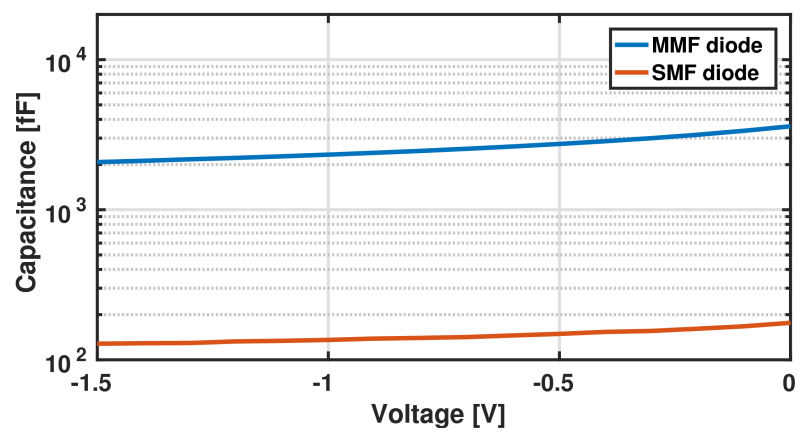

(b)

Fig. 8: (a) Diode impedances extracted from s-parameter measurements for different biasing voltages. (b) Diode junction capacitances for different biasing voltages.

The SMF diode has a capacitance of $175-125 \mathrm{fF}$ for reverse biasing voltages of $0-1.5 \mathrm{~V}$, while the MMF diode junction capacitance ranges between $3.5-2 \mathrm{pF}$ for the same biasing

\begin{tabular}{c|c|c}
\hline Spec & 40nm N-well diode [9] & 28nm N-well diode \\
\hline Schottky Barrier [eV] & 0.55 & 0.7 \\
Built-in Potential [V] & 0.65 & 0.69 \\
C/A [mF/m ${ }^{2}$ ] & 4.8 & 1.7 \\
850nm Resp. [A/W] & N/A & $0.27 \mathrm{e}-3$ (FSI) \\
1310nm Resp. [A/W] & $0.4 \mathrm{e}-3$ (BSI) & $5.8 \mathrm{e}-6$ (FSI), 0.71e-3 (BSI) \\
1550nm Resp. [A/W] & N/A & $1.1 \mathrm{e}-6$ (FSI), $0.16 \mathrm{e}-3$ (BSI) \\
\hline
\end{tabular}

TABLE I: Summary measurements results

voltages. This capacitance-to-voltage relationship reveals that the built-in potential of the Schottky diode is $0.69 \mathrm{~V}$. At zero bias, Schottky diodes in this technology have a capacitance-toarea ratio of $1.7 \mathrm{mF} / \mathrm{m}^{2}$, whereas the diodes in [9] processed in $40 \mathrm{~nm}$ CMOS have a ratio of $4.8 \mathrm{mF} / \mathrm{m}^{2}$. As the builtin potential of that diode is $0.65 \mathrm{~V}$, this capacitance-to-area ratio also implies the $\mathrm{N}$-well doping in that technology is higher. While Schottky diodes in this technology achieve a lower responsivity due to the higher Schottky barrier, the lower capacitance in turn relaxes the specs of the analog front-end [4]. The measurement results are summarized in TABLE I.

\section{Conclusion}

$\mathrm{N}$-well Schottky diodes in standard 28nm CMOS technology have been presented. These diodes can convert a very large spectrum of light to electric current at a large intrinsic bandwidth. Measurement results of I-V characteristics, capacitance and DC responsivity of 850,1310 and $1550 \mathrm{~nm}$ light have been shown. This is the first reported $1550 \mathrm{~nm}$ responsivity of a CMOS photodetector. In comparison to Nwell Schottky diodes in 40nm CMOS technology, the diodes in this technology achieve lower responsivity due to a higher Schottky barrier, but a lower capacitance-to-area ratio.

\section{REFERENCES}

[1] E. Säckinger, Broadband Circuits for Optical Fiber Communication. Wiley, 2005.

[2] A. Rahim et al., "Expanding the silicon photonics portfolio with silicon nitride photonic integrated circuits," OSA $J L T$, vol. 35, no. 4, pp. 639649, Feb 2017.

[3] Y. A. Vlasov, "Silicon cmos-integrated nano-photonics for computer and data communications beyond 100g," IEEE Communications Magazine, vol. 50, no. 2, pp. s67-s72, February 2012.

[4] D. Lee, J. Han, G. Han, and S. M. Park, "An 8.5-gb/s fully integrated cmos optoelectronic receiver using slope-detection adaptive equalizer," IEEE JSSC, vol. 45, no. 12, pp. 2861-2873, Dec 2010.

[5] S. H. Huang, W. Z. Chen, Y. W. Chang, and Y. T. Huang, "A 10-gb/s oeic with meshed spatially-modulated photo detector in 0.18-um cmos technology," IEEE JSSC, vol. 46, no. 5, pp. 1158-1169, May 2011.

[6] J. S. Youn, M. J. Lee, K. Y. Park, and W. Y. Choi, "10-gb/s 850-nm cmos oeic receiver with a silicon avalanche photodetector," IEEE QE, vol. 48, no. 2, pp. 229-236, Feb 2012.

[7] J. Genoe, D. Coppee, J. Stiens, R. A. Vonekx, and M. Kuijk, "Calculation of the current response of the spatially modulated light cmos detector," IEEE ED, vol. 48, no. 9, pp. 1892-1902, Sep 2001.

[8] M. M. Hayat, B. E. A. Saleh, and M. C. Teich, "Effect of dead space on gain and noise of double-carrier-multiplication avalanche photodiodes," IEEE ED, vol. 39, no. 3, pp. 546-552, Mar 1992.

[9] W. Diels, M. Steyaert, and F. Tavernier, "Schottky diodes in 40nm bulk cmos for 1310nm high-speed optical receivers," in 2017 Optical Fiber Communications Conference and Exhibition, March 2017, pp. 1-3.

[10] S. M. Sze and K. K. Ng, Physics of Semiconductor Devices, 3rd ed. Wiley-Interscience, 2006.

[11] R. Han et al., "A 280-ghz schottky diode detector in 130-nm digital cmos," IEEE JSSC, vol. 46, no. 11, pp. 2602-2612, Nov 2011. 